\title{
Scheduling for non regular performance measure under the due window approach
}

\author{
Ihsan Sabuncuoglu*, Tahar Lejmi \\ Department of Industrial Engineering, Bilkent University, 06533 Bilkent, Ankara, Turkey
}

Received 1 April 1998; accepted 1 February 1999

\begin{abstract}
In the last two decades, Just-In-Time (JIT) production has proved to be an essential requirement of world class manufacturing. This has made schedulers most concerned about the realization of a JIT environment. The JIT concept requires not only a penalty for backorder and lateness but also for earliness. This can be translated into non-regular scheduling objectives. The most obvious objective can be to minimize the deviation of completion times. Concerning earliness/tardiness problems, researchers have usually considered systems where jobs incur no penalty for completion at a certain point of time (i.e. due date). In practice, however, job completions can also be accepted without penalty within an interval in time, which is known as the due window. This paper studies the scheduling problems in terms of the non-regular measure, mean absolute deviation (MAD), under the due window approach. The study is conducted in a dynamic job shop environment. Furthermore, we propose two new rules that perform quite effectively for the MAD measure. (C) 1999 Elsevier Science Ltd. All rights reserved.
\end{abstract}

Keywords: Job shop scheduling; Due windows; MAD

\section{Introduction}

Due to the tremendous increase in international competition in the last two decades, Just-In-Time (JIT) production has proved to be an essential requirement of world class manufacturing. The JIT philosophy seeks to identify and eliminate waste components as over production, waiting time, transportation, processing, inventory, movement, and defective products [10]. Consequently, it is important that the area of scheduling contribute towards the realization of a JIT environment.

\footnotetext{
* Corresponding author. Tel.: +90-312-266-4477; fax: +90312-266-4126.

E-mail address: sabun@bilkent.edu.tr (I. Sabuncuoglu)
}

For many years, scheduling research focused on single performance measures, referred to as regulars measure, that are non decreasing in job completion times [3]. Most of the literature deals with regular measures such as mean flow time, mean lateness, percentage of jobs tardy, and mean tardiness. In particular, the mean tardiness criterion has been a standard way of measuring conformance to due dates, although it ignores the consequences of jobs completing early. However, this emphasis has changed with the current interest in JIT production. The JIT concept requires not only a penalty for backorder and lateness but also for earliness [10]. Therefore, an ideal schedule is one in which all jobs finish exactly on their assigned due dates. This can be translated to a non-regular scheduling objective. The most obvious objective is to minimize the deviation of completion times [3]. However, 
there are other ways to measure the goodness of a schedule. Readers can refer to [3] for a review of non regular earliness/tardiness objectives.

The theoretical model of just in time scheduling assumes only one point in time is an acceptable completion time and any earliness or tardiness is penalized. However, in manufacturing industries, a due date is often considered as an interval of time rather than a single point in time [10]. Namely, for each job to be processed on the machine, there is an earliest due date and a latest due date. Any job finished after its latest due date is considered tardy. No job can be delivered before its earliest due date. It must be held until its earliest due date if it finishes earlier and hence it incurs an inventory cost. The time period between its earliest and latest due date is called the due window. A job finished within its due window does not incur any penalty.

In this paper, we will extend the earliness and tardiness measure from the single due date case to the due window case. In fact, recent research in this area has dealt mainly with static scheduling. In other words, the set of jobs to be scheduled is known in advance and is simultaneously available. In this section, we analyze the problem in a dynamic environment. Specifically, we test the performance of several well-known priority rules in a dynamic job-shop for an earliness-tardiness measure via simulation.

Many articles dealing with due window problems suggested MAD (mean absolute deviation from job completion times) as an appropriate non regular measure for earliness-tardiness problems [15]. We also use MAD for dynamic scheduling with some common priority rules. In this paper, we also propose two new rules for the MAD measure. The preliminary tests indicate that the proposed rules are quite effective in reducing MAD.

The rest of the paper is organized as follows. Section 2 presents both the new rules and the existing priority rules used in the study. Section 3 gives the system considerations and experimental conditions. Section 4 illustrates the modeling procedure of due windows and describes the implementation of our model. Analyses of the results are presented in section 5 . Finally, the paper ends with concluding remarks in section 6

\section{Scheduling rules}

\subsection{Priority rules included in the study}

According to Kiran and Smith [9] and Baker and Kanet [2], SPT and MOD rules are the most effective non parameterised rules for completion time and tardiness based criteria, respectively. In this paper, we try
Table 1

Mathematical description of priority rules used in the study

Priority rules Mathematical description

\begin{tabular}{lll}
\hline Processing time based & SPT & $P_{i j}{ }^{\mathrm{a}}$ \\
& STPT & $P_{i}$ \\
Simple rules & FCFS & $r_{i j}$ \\
& FAFS & $R_{i}$ \\
Due date based rules & EDD & $D_{i}$ \\
& ODD & $d_{i j}=R_{i}+\frac{D_{i}-R_{i}}{P_{i}} \sum_{q=1}^{i} p_{i q}$ \\
& JSLACK & $D_{i}-t-P_{i j}$ \\
& OSLACK & $d_{i j}-t-p_{i j}$ \\
& MOD & $\operatorname{Max}\left(d_{i j}, t+p_{i j}\right)$ \\
& MDD & $\operatorname{Max}\left(D_{i}, t+P_{i j}\right)$
\end{tabular}

${ }^{\text {a }} i$ Index for job $i ; j$ Index for operation $j ; D_{i}$ Due date of job $i$; $d_{i j}$ Due date of job $i$ for operation $j ; R_{i}$ Arrival time of job $i$ at the system; $r_{i j}$ Ready time of job $i$ at operation $j ; P_{i}$ Total operation time for job $i$; $P_{i j}$ Total remaining processing time for job $i ; p_{i j}$ Processing time of job $i$ at operation $j ; t$ Time index.

to find out whether these rules are also effective with the MAD measure. Note that SPT and MOD are described as local rules. Conway and Maxwell [5] define local priority rules as those that require information only about those jobs that are waiting at a machine, while global rules require additional information about jobs or machine states or other machines. Shortest total processing time (STPT) and the modified job due date (MDD) rules can be considered as the global rules in this context. In this study we use these four rules. To seek more generality, we use three other local/global pairs of rules: ODD and EDD, OSLACK and JSLACK, which are again proved to be simple but very effective rules. FCFS and FAFS rules are included in the study as the benchmark rules. Table 1 gives the mathematical definitions for these eight rules selected.

\subsection{Two new rules developed for MAD}

\subsubsection{Background: Review of $E / T$ problems with $M A D$}

The existing studies in the literature on $E / T$ (Earliness/Tardiness) problems deals with static scheduling i.e., the set of jobs to be scheduled is known in advance and is simultaneously available. The vast majority of the articles on E/T problems deal with singlemachine models. In addition, in all these studies, the objective is usually to minimize the total penalty cost. However, as indicated by Baker and Scudder [3], the penalties can be measured in different ways.

An important class in the family of $\mathrm{E} / \mathrm{T}$ problems involves minimizing the sum of absolute deviations of the job completion times from a common due date $d$ (i.e. MAD with common due date $d$ for all jobs). The 
analysis of this problem is due to Kanet [8], Sundararaghvan and Ahmet [13], Hall [7] and Bagchi, Chang and Sullivan [1]. A detailed summary is also given by Emmons [6]. As the work done by Baker and Scudder [3] indicates, the solution to the problem can be described qualitatively. It is desirable to construct the schedule so that the due date is, in some sense, in the middle of the jobs. Baker and Scudder [3] state that, for a relatively loose common due date, there exists an optimal schedule to the unrestricted problem with the following property; the optimal schedule is $\mathrm{V}$ shaped. That is jobs that have their completion times: $C_{j} \leq d$ are sequenced in non increasing order of processing times (i.e. according to LPT, longest processing time first rule); jobs for which $C_{j}>d$ are sequenced in non decreasing order of processing time (i.e. according to the SPT rule). This property (property II in their paper) implies that once the membership in the two sets is known, the sequence of the jobs within each set can be determined using LPT and SPT rules. This means that a solution can be partitioned into two sets of jobs, an early set and a tardy set. Baker and Scudder [3] suggest an algorithm of order $O(n \log n)$ to find out the tardy and early sets leading to an optimal schedule for the problem.

\subsubsection{Derivation of two new rules}

From the above discussion, one might get an impression that the algorithm suggested by Baker and Scudder to the single machine/common due date problem can serve as a basis to construct heuristics or priority rules for MAD in the dynamic job shop environment. However, it only suggests a procedure to identify the set of early and tardy sets mentioned above, given that the size of the total job population is known in advance. Nevertheless, in a dynamic environment where the number of jobs arriving to a certain machine vary over time, it may be a very difficult task to apply such a procedure. As a result of our analysis, we propose two alternative methods to differentiate early and tardy jobs. Both methods are similar in nature, but one uses local information whereas the other uses global information. The idea is to assign to every arriving job at a certain machine, an index value that indicates whether that job is expected to be tardy or early. The index is built according to either global or local job information as follows:

$I_{1}=d_{j k}-c_{j k}$ and $I_{\mathrm{g}}=D_{j}-C_{j}$

where $I_{1}$ : Local index, $I_{\mathrm{g}}$ : Global index, $c_{j k}=p_{j k}+t$ : Estimated completion time of operation $k$ of job $j$, where $p_{j k}$ is the operation processing time and $t$ is the index for current time, $d_{j k}$ : Operation $k$ due date of job $j, D_{j}$ : Job $j$ due date, $C_{j}=P_{j}+t$ : Job $j$ estimated com- pletion time, where $P_{j}$ is to total remaining job processing time.

Clearly, when $I_{1}<0$ or $I_{\mathrm{g}}<0$, it means that the job is locally or globally tardy respectively. Inversely, when $I_{1}>0$ or $I_{\mathrm{g}}>0$, then the job is early. Accordingly, the membership to early and tardy sets is defined. In fact, these indexes are known in the literature as operation and job slack respectively. Hence, for convenience, we will use these terms instead of local and global indexes. Now a straightforward implication of Baker and Scudder's V-shaped scheduling policy, is the fact that, once the membership of the jobs is defined (Tardy or Early), the jobs are ranked according to LPT or SPT order, respectively. This suggests two rules, named as NOS (Normalized Operation Slack) and NJS (Normalized Job Slack), that select the jobs according to the minimum of $\pi_{1}$ and $\pi_{2}$ defined below:

$$
\begin{aligned}
& \text { NOS: } \pi_{1}=\frac{d_{j k}-t-p_{j k}}{\left|d_{j k}-t-p_{j k}\right|} \times \frac{1}{p_{j k}} \\
& \text { NJS: } \pi_{2}=\frac{D_{j}-t-P_{j}}{\left|D_{j}-t-P_{j}\right|} \times \frac{1}{p_{j k}}
\end{aligned}
$$

The above rules assign the priorities in ascending order of $l / p_{j k}$ (LPT), and $-l / p_{j k}$ (SPT) for negative, positive operation/job slack jobs, respectively. In fact, we could have satisfied ourselves by these two rules, but, as it will be seen later, these rules are no better than the traditional rules in most of the experimental conditions. Intuitively, this is due to the fact that such rules ignore the dynamic aspect of our job shop system, where job slacks are different from one job to another, and vary over time. Consequently, we had high expectancies that if we also consider the magnitude of the slack while prioritizing jobs, this would improve the performance of the queuing policy. Consequently, we suggest that we simply prioritize jobs according to the product of the job/operation slacks and $l / p_{j k}$. In other words, the priority is given based on the confounding effect of the slack and processing times. Note that, for equal positive slack jobs, multiplying by $l / p_{j k}$ ensures that the jobs are ordered in LPT order. However, for equal negative slack jobs, a similar multiplication ensures that the jobs follow the SPT order. Now our two rules would select the jobs according to the minimum of $\pi_{3}$ and $\pi_{4}$, which are defined as follows:

Rule 1: $\pi_{3}=\frac{d_{j k}-t-p_{j k}}{p_{j k}}$

Rule 2: $\pi_{4}=\frac{D_{j}-t-P_{j}}{p_{j k}}$

To enhance the efficiency of the above rules under the 
due window approach, we restructure them so that they use two pieces of due date information rather than one information at a time. The final expression of the rules, that we will name as MOS (modified operation slack) and MJS (modified job slack), is as follows:

MOS:

$$
\pi_{3}=\frac{\max \left(d_{j k}^{1}-t-p_{j k}, 0\right)+\min \left(d_{j k}^{\mathrm{e}}-t-p_{j k}, 0\right)}{p_{j k}}
$$

MJS: $\pi_{4}=\frac{\max \left(D_{j}^{1}-t-P_{j}, 0\right)+\min \left(D_{j}^{\mathrm{e}}-t-P_{j}, 0\right)}{p_{j k}}$

where, $d_{j k}^{\mathrm{e}}, D_{j}^{\mathrm{e}}, d_{j k}^{\mathrm{l}}$ and $D_{j}^{1}$ are the earliest/latest operation/job due dates respectively.

MOS rule works as follows: Given a certain job $j$ with operation $k$, the rule considers job $j$ as early if its estimated operation completion time $c_{j k}\left(=p_{j k}+t\right)$ is less than its earliest operation due date $d_{j k}^{\mathrm{e}}$. Conversely, it assumes that job $j$ belongs to the set of tardy jobs if $c_{j k} \geq d_{j k}^{l}$, where $d_{j k}^{d}$ is its latest operation due date. More importantly, when $d_{j k}^{e} \leq \mathrm{c}_{j k} \leq d_{j k}^{i}$, the rule considers the job as tardy or early depending on how close its completion time $\left(c_{j k}\right)$ is to the earliest and latest due dates. That is, if $c_{j k}$ is closer to $d_{j k}^{\mathrm{e}}$ than to $d_{j k}^{\mathrm{i}}$, than job $j$ is presumed early, otherwise, tardy. The MJS rule works in a similar way, but it uses job rather than operation based due date and completion time information.

As a matter of fact, within further analysis, we believe that it is more reasonable to focus mainly on MOS and MJS, while keeping only a supportive role for NJS and NOS rules.

\section{System considerations, simulation model and experimental conditions}

\subsection{Suggested model}

In a dynamic and stochastic manufacturing environment, testing scheduling rules under different experimental conditions becomes a more complex task than in the static case. It follows from the fact that one should be very careful on the model choice. The generality aspect of such a model must be kept at maximum in order to get potential benefits from the experiments.

Our model is similar to the one used by Vepsalainen and Morton [14]. It is a re-entrant dynamic job shop model with:

- Continuously available 10 machines

- Continuous arrival of jobs having a Poisson distribution
- Number of operations assigned to each job arrived is random having a uniform distribution $\mathrm{U}(1,10)$

- Each operation is equally likely to be performed on ten machines, where processing times are random having a uniform distribution $\mathrm{U}(1,30)$.

The assumptions of this model are given in the study by Vepsalainen and Morton [14].

\subsection{Experimental conditions}

\subsubsection{System load (or machine and shop utilization)}

The combined effects of job arrival distribution, job routing and processing times determine system load (or the machine utilization). From the standpoint of job-shop simulation, machine utilization is important because it affects queue lengths. If the average queue length is too small, the scheduling rules used in the model may not be forced to make discriminating job selections; when this situation occurs, an evaluation of rule effectiveness is difficult or impossible. Adverse effects also result from machine utilization when it is too high. If utilization is near $100 \%$, transient conditions may extend over a long time period. Machine utilization commonly found in the literature ranges from 60 to $95 \%$. This range of utilization permits scheduling rules to select a job from several in the queue but does not lead to very long queues. In this paper, we consider two levels of machine utilization: $60 \%$ (low) and $85 \%$ (high). We achieve the desired utilization level by adjusting the arrival rate.

\subsubsection{Due date tightness and assignment rule}

Due date performance of the rules is affected by due date tightness. In general, tighter due dates tend to produce larger values of MT (mean tardiness) and PT (proportion of tardy jobs), if other conditions remain unchanged [4]. Beyond that there is also evidence that the relative performance of priority rules is also affected by due date tightness, at least for PT and for MT. This suggests the existence of so called cross over points, with one rule performing best for tighter due dates and another performing best for looser due dates. In this study, we use the TWK approach in assigning the due dates. The reason is that TWK method is found to be the most efficient rule to reduce the cross over effect [4]. According to TWK, job due dates are defined as follows:

$D_{j}=R_{j}+A_{j}$

where,

$A_{j}=k \times P_{j}$ represents the original flow allowance,

$k$ is the due date tightness value, and

$R_{j}$ denotes the arrival time of $j$.

Baker suggests that $10 \%$ and $40 \%$ PT values represent loose and relatively tight due dates respectively. 
These PT values are used as reference values to apply due date tightness to the simulation experiments in almost all the study, except that, in light of the symmetric criterion MAD, we lately perform some experiments with the extremely tight due dates case. In order to set tight due dates or loose due dates, the parameter $k$ should be adjusted so that we achieve the required PT values mentioned previously (i.e. $10 \%$ and $40 \%$ values). In this study, we use separate pilot runs to set the values of $k$, with respect to FCFS (benchmark rule) for different machine utilization levels. Note that the same value of $\mathrm{k}$ is used for all tested priority rules under the same utilization level.

\subsubsection{Performance criteria}

In this study we deal with a non regular measure, the mean absolute deviation (MAD) criterion. Note that MAD is the sum of two other well known criteria, mean earliness (ME) and mean tardiness (MT), that will be also measured as they are useful to the analysis later. This will be justified in the next section. Note that also, the performance of the rules in terms of the latter two criteria is also measured in the experiments.

\section{Modeling due windows}

In practice, a job due date can be assigned as a time interval (due window) rather than a point in time. Specifically, for each job to be processed on the machine, there can be an earliest due date and a latest due date. Any job finished after its latest due date is considered tardy. No job can be delivered before its earliest due date, it must be held until its delivery time. Krämer and Lee [10] define a due window as the time interval limited by the latest and earliest due dates for a given job in a manufacturing environment. In our study, we will use a simple approach that will be defined as follows:

Each job entering the system will be given a certain due date $D_{j}$ using TWK method. Earliest and latest job due dates are defined respectively as follows:

$D_{j}^{\mathrm{e}}=D_{j}-R \times A_{j}^{\mathrm{o}}$

$D_{j}^{1}=D_{j}+R \times A_{j}^{\mathrm{o}}$

where

$D_{j}^{\mathrm{e}}$ : is the earliest due date for job $j$.

$D_{j}^{1}$ : is the latest due date for job $j$.

$R$ : is the radius coefficient of the interval.

$A_{j}^{\mathrm{o}}$ : is the flow allowance assigned to job $j$ at time zero.

The radius coefficient $R$ is initially set to $10 \%$. Later, we use different values of $R(0,20$ and $40 \%)$ in

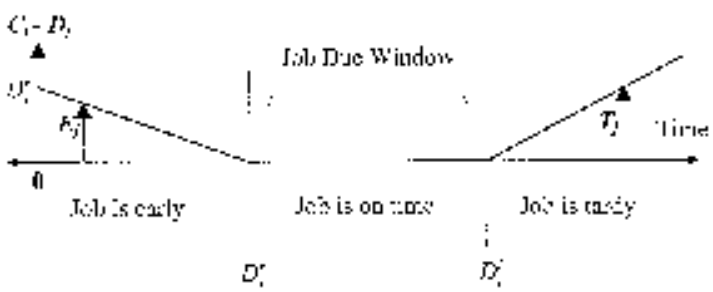

Fig. 1. Illustration of $E_{j}$ and $T_{j}$ under the due window approach.

order to compare the relative performance of MOS and MJS with the most competing rule MOD, under different due window sizes.

Now, recalling that MOS is a local information based rule, it is necessary to define the earliest and latest operation due dates, that are constructed according to the TWK method. Their definition is as follows:

$d_{i j}^{\mathrm{e}}=R_{i}+\frac{D_{i}^{\mathrm{e}}-R_{i}}{P_{i}} \sum_{q=1}^{i} p_{i q}$

$d_{i j}^{\mathrm{d}}=R_{i}+\frac{D_{i}^{1}-R_{i}}{P_{i}} \sum_{q=1}^{i} p_{i q}$

where

$d_{i j}^{\mathrm{e}}, D_{i}^{\mathrm{e}}, d_{i j}^{1}$ and $D_{i}^{1}$ are the earliest/latest operation/job due dates respectively

$R_{i}=$ Arrival time of job $i$ at the system

$r_{i j}=$ Ready time of job $i$ at operation $j$

$P_{i}=$ Total operation time for job $i$

$P_{i j}=$ Total remaining processing time for job $i$

$p_{i j}=$ Processing time of job $i$ at operation $j$

A point to note here is that when implementing the due window approach, one is faced with the problem of choosing the due date information that should be used by due date based priority rules (i.e. EDD, ODD, MDD, MOD, JSLACK, OSLACK, NJS, and NOS). Hence, in the simulation experiments, we test the rules with three due date information categories: earliest due date $\left(D_{j}^{\mathrm{e}}\right)$, original due date $\left(D_{j}\right)$ and latest due date $\left(D_{j}^{1}\right)$.

Now, according to the above definition of earliest and latest due dates, MAD, ME (mean earliness) and MT (mean tardiness) are expressed as follows:

$\mathrm{MAD}=\frac{\sum_{j=1}^{n}\left(E_{j}=T_{j}\right)}{n}, \mathrm{ME}=\frac{\sum_{j+1}^{n} E_{j}}{n}, \mathrm{MT}=\frac{\sum_{j=1}^{n} T_{j}}{n}$

where,

$E_{j}=\operatorname{Max}\left(D_{j}^{\mathrm{e}}-C_{j}, 0\right)$ : Earliness of job $j$

$T_{j}=\operatorname{Max}\left(C_{j}-D_{j}^{1}, 0\right)$ : Tardiness of job $j$ 
Table 2

Due date tightness parameter $k$ values

\begin{tabular}{lll}
\hline & Tight due dates & Loose due dates \\
\hline $\begin{array}{l}\text { High machine } \\
\text { utilisation }(85 \%)\end{array}$ & $k=3.8$ & $k=6.5$ \\
$\begin{array}{l}\text { Low machine } \\
\text { utilisation }(60 \%)\end{array}$ & $k=1.8$ & $k=2.7$ \\
\hline
\end{tabular}

In Fig. 1, we illustrate graphically $E_{j}$ and $T_{j}$ under the due window approach.

\subsection{Model implementation}

The simulation models are developed using the SIMAN language [12]. The common random number variance reduction technique $(\mathrm{CRN})$ is implemented to compare the rules under identical conditions and to reduce the experimental error. Initially, some pilot runs are taken to find suitable values of the arrival rates to set the desired utilization levels. Two values are found for the arrival rate: 10.3 and 14.5 corresponding to 85 and $60 \%$ utilization levels, respectively. Furthermore, several other runs are also taken to estimate the warm up period using the Welch approach [11]. As a result, 300 job completions are deleted at the beginning of each run to reduce the effect of initial bias. In order not to lose too much computer time, the batch means approach is used, ten batches are analyzed for each experiment run, with a batch size equals 900 observations in each run. Pilot runs are also taken to set the parameter $k$, with respect to FCFS for each machine utilization level as shown in Table 2.

\section{Computational results}

\subsection{Results of traditional rules}

The results of the simulation experiments are collectively given in Tables 3 and 4. In Table 3, we illustrate the performance of the rules for ME, MT and MAD (refer to Eqs. 3 on p. 12) with respect to each due date information category used. The table includes three major columns, each corresponding to one due date information category. Within each major column, you can find three minor columns corresponding to ME, MT, and MAD criteria respectively. Note also that the results for the traditional and new rules are stated separately within the tables. Initially, we analyze the performance of the existing rules. The pair $t$-tests are applied to measure the statistical significance of the difference between the best two performances, only among the traditional eight rules. The sign $\left(^{*}\right)$ indicates that the difference is significant $\alpha=0.05$ level. Later, we measure the performance of the new rules: NJS, NOS, MJS, and MOS with main focus on the latter two rules, that are developed in this study for MAD. The pair $t$-tests are applied again to measure the statistical significance of the difference between the performance of the best of our two new rules and the best performing traditional rule. The sign (") indicates that the difference is significant at $\alpha=0.05$ level.

From Table 3, it is obvious that the performance of the rules is quite sensitive to two main experimental factors: due date tightness and machine utilization. For instance, with reference to the first part of Table 3 , we can observe at high machine utilization level $(85 \%)$ that the performance of the rules has improved on the average by $60 \%$ as due dates get tighter. This infers a strong positive correlation between the degree of tightness of due dates and the MAD performance of the rules. This correlation could be explained by the fact that the rules tend to produce less early jobs (i.e. lower ME) when due dates get tighter. On the other hand, as one can intuitively expect, the performance of the rules improves as the system load decreases.

According to Table 3, we observe that SPT and STPT, which are known to be the best for MF (mean flow time $=$ average of the completion times), perform very poorly for MAD. As can be noted, even the benchmark rules FCFS and FAFS display better performance than SPT and STPT. This indicates that SPT and STPT are not appropriate rules to minimize MAD because these rules which seek primarily to minimize job completion times, have the tendency to produce very early jobs and hence result in high MAD values.

In contrast, due date based rules show better MAD performances than any non due date based rule under all experimental conditions. In general, MOD displays the best MAD performances than any other competing rule under all but the low utilization case. OSLACK shows the second best performance and is followed by ODD, JSLACK, EDD and MDD.

A point worth noting is that the rules (both non due date and due date base rules) produce relatively high MAD values in the loose due dates case because of too many early job completions. This suggests looking for new rules that are more effective in the loose due dates' case.

In Table 4, we measure the variability of the prediction error by calculating the standard deviation of the earliness, tardiness, and absolute deviation performances of the rules as illustrated in the table. We mainly conclude from the results that STPT has the least variability when the system is highly loaded, whereas MOD is the best rule in the low utilization case.

\subsubsection{Rule's sensitivity to the due date information}

In order to analyze the sensitivity of the rules to the 
Table 3

ME, MT and MAD simulation results under due window approach

\begin{tabular}{|c|c|c|c|c|c|c|c|c|c|}
\hline & \multicolumn{3}{|c|}{ Earliest due datate } & \multicolumn{3}{|c|}{ Original due date } & \multicolumn{3}{|c|}{ Latest due date } \\
\hline & ME & MT & MAD & ME & MT & MAD & ME & MT & MAD \\
\hline \multicolumn{10}{|c|}{ High utilisation $(85 \%) /$ Tight due dates $(k=3.8)$} \\
\hline SPT & 84.36 & 41.74 & 126.10 & 84.36 & 41.74 & 126.10 & 84.36 & 41.74 & 126.10 \\
\hline STPT & 79.70 & 79.25 & 158.95 & 79.70 & 79.25 & 158.95 & 79.70 & 79.25 & 158.95 \\
\hline FCFS & 34.51 & 54.91 & 89.42 & 34.51 & 54.91 & 89.42 & 34.51 & 54.91 & 89.42 \\
\hline FAFS & 38.16 & 56.13 & 94.29 & 38.16 & 56.13 & 94.29 & 38.16 & 56.13 & 94.29 \\
\hline EDD & 30.63 & 28.60 & 59.23 & 31.83 & 29.69 & 61.52 & 33.75 & 29.62 & 63.37 \\
\hline ODD & 30.66 & 25.44 & 56.10 & 31.38 & 23.79 & 55.16 & 32.55 & 24.95 & 57.50 \\
\hline JSLACK & 28.07 & 29.47 & 57.55 & 30.15 & 27.00 & 57.15 & 30.74 & 28.59 & 59.32 \\
\hline OSLACK & 28.03 & 28.48 & 56.52 & 29.70 & 24.65 & 54.36 & 30.69 & 26.49 & 57.18 \\
\hline MOD & 39.35 & $23.76^{*}$ & 63.11 & 36.73 & $20.44^{*}$ & 57.18 & 35.61 & $17.30^{*}$ & $52.91^{*}$ \\
\hline MDD & 34.87 & 29.80 & 64.66 & 33.46 & 28.57 & 62.03 & 33.25 & 28.51 & 61.76 \\
\hline \multicolumn{10}{|l|}{ New rules } \\
\hline NJS & $19.61^{\prime \prime}$ & 28.69 & 48.30 & $21.70^{\prime \prime}$ & 32.81 & 54.51 & 23.41 & 44.70 & 68.10 \\
\hline NOS & 30.78 & 26.73 & 57.52 & 28.50 & 26.09 & 54.59 & 27.86 & 26.95 & 54.80 \\
\hline MJS & 21.72 & 31.95 & 53.68 & 21.72 & 31.95 & 53.68 & $21.72^{\prime \prime}$ & 31.95 & 53.68 \\
\hline MOS & 25.29 & $19.37^{\prime \prime}$ & $44.67^{\prime \prime}$ & 25.29 & 19.37 & $44.67^{\prime \prime}$ & 25.29 & 19.37 & $44.67^{\prime \prime}$ \\
\hline \multicolumn{10}{|c|}{ High utilisation $(85 \%) /$ Loose due dates $(k=6.5)$} \\
\hline SPT & 264.22 & 19.62 & 283.85 & 264.22 & 19.62 & 283.85 & 264.22 & 19.62 & 283.85 \\
\hline STPT & 242.37 & 38.74 & 281.11 & 242.37 & 38.74 & 281.11 & 242.37 & 38.74 & 281.11 \\
\hline FCFS & $178.3^{*}$ & 10.26 & 188.64 & $178.3^{*}$ & 10.26 & 188.64 & $178.3^{*}$ & 10.26 & 188.64 \\
\hline FAFS & 192.35 & 19.12 & 211.47 & 192.35 & 19.12 & 211.47 & 192.35 & 19.12 & 211.47 \\
\hline EDD & 186.02 & 0.29 & 186.31 & 192.26 & 0.20 & 192.46 & 187.36 & 0.29 & 187.66 \\
\hline ODD & 190.53 & 0.58 & 191.11 & 190.00 & 0.34 & 190.34 & 189.24 & 0.35 & 189.58 \\
\hline JSLACK & 182.06 & 0.29 & 182.35 & 184.77 & $0.14^{*}$ & 184.91 & 186.98 & $0.14^{*}$ & 187.12 \\
\hline OSLACK & 187.03 & 0.72 & 187.75 & 184.16 & 0.55 & 184.70 & 185.66 & 0.48 & 186.15 \\
\hline MOD & 190.89 & 1.55 & 192.45 & 188.85 & 0.65 & 189.50 & 189.49 & 0.42 & 189.91 \\
\hline MDD & 187.55 & 0.82 & 188.37 & 188.98 & 0.25 & 189.23 & 190.22 & 0.33 & 190.55 \\
\hline \multicolumn{10}{|l|}{ New rules } \\
\hline NJS & $118.0^{\prime \prime}$ & 1.52 & $119.5^{\prime \prime}$ & 125.64 & 3.89 & 129.53 & 128.71 & 14.73 & 143.44 \\
\hline NOS & 143.02 & 2.95 & 145.97 & 141.03 & 2.48 & 143.50 & 138.33 & 4.40 & 142.73 \\
\hline MJS & 125.1 & 0.59 & 125.7 & $125.1^{\prime \prime}$ & 0.59 & $125.7^{\prime \prime}$ & $125.1^{\prime \prime}$ & 0.59 & $125.7^{\prime \prime}$ \\
\hline MOS & 146.10 & 0.68 & 146.75 & 146.07 & 0.68 & 146.75 & 146.07 & 0.68 & 146.75 \\
\hline \multicolumn{10}{|c|}{ Low utilisation $(60 \%) /$ Tight due dates $(k=1.8)$} \\
\hline SPT & 12.63 & 12.98 & 25.62 & 12.63 & 12.98 & 25.62 & 12.63 & 12.98 & 25.62 \\
\hline STPT & 11.65 & 19.96 & 31.62 & 11.65 & 19.96 & 31.62 & 11.65 & 19.96 & 31.62 \\
\hline FCFS & 9.18 & 20.56 & 29.75 & 9.18 & 20.56 & 29.75 & 9.18 & 20.56 & 29.75 \\
\hline FAFS & 10.37 & 21.62 & 31.99 & 10.37 & 21.62 & 31.99 & 10.37 & 21.62 & 31.99 \\
\hline EDD & 9.36 & 15.33 & 24.69 & 9.47 & 15.76 & 25.23 & 9.41 & 15.16 & 24.57 \\
\hline ODD & 8.19 & 13.74 & 21.93 & 8.43 & 12.88 & 21.31 & 8.66 & 12.49 & 21.14 \\
\hline JSLACK & 8.12 & 15.95 & 24.08 & 8.25 & 15.05 & 23.30 & 8.52 & 14.97 & 23.49 \\
\hline OSLACK & 7.89 & 16.26 & 24.15 & 8.22 & 15.54 & 23.76 & 8.24 & 14.42 & 22.66 \\
\hline MOD & 9.79 & $11.25^{*}$ & 21.04 & 9.21 & $10.61^{*}$ & 19.80 & 9.05 & $10.37^{*}$ & $19.43^{*}$ \\
\hline MDD & 9.83 & 17.84 & 27.66 & 9.64 & 16.51 & 26.15 & 9.46 & 15.46 & 24.92 \\
\hline \multicolumn{10}{|l|}{ New rules } \\
\hline NJS & 8.28 & 13.84 & 22.12 & 8.52 & 15.75 & 24.28 & 9.06 & 18.72 & 27.78 \\
\hline NOS & 8.97 & 11.43 & 20.40 & 8.72 & 11.57 & 20.29 & 8.92 & 12.59 & 21.51 \\
\hline MJS & 8.05 & 15.01 & 23.06 & $8.05^{\prime \prime}$ & 15.01 & 23.06 & $8.05^{\prime \prime}$ & 15.01 & 23.06 \\
\hline MOS & 8.20 & 11.80 & $20.00^{\prime \prime}$ & 8.20 & 11.80 & 20.00 & 8.20 & 11.80 & 20.00 \\
\hline \multicolumn{10}{|c|}{ Low utilisation $(60 \%) /$ Loose due dates $(k=2.7)$} \\
\hline SPT & 64.89 & 3.58 & 68.47 & 64.89 & 3.58 & 68.47 & 64.89 & 3.58 & 68.47 \\
\hline STPT & 59.05 & 5.91 & 64.96 & 59.05 & 5.91 & 64.96 & 59.05 & 5.91 & 64.96 \\
\hline FCFS & 52.75 & 4.70 & 57.46 & 52.75 & 4.70 & 57.46 & 52.75 & 4.70 & 57.46 \\
\hline FAFS & 56.37 & 6.92 & 63.29 & 56.37 & 6.92 & 63.29 & 56.37 & 6.92 & 63.29 \\
\hline EDD & 53.54 & 1.24 & 54.78 & 53.74 & 1.41 & 55.16 & 54.01 & 1.33 & 55.34 \\
\hline
\end{tabular}


Table 3 (continued)

\begin{tabular}{|c|c|c|c|c|c|c|c|c|c|}
\hline & \multicolumn{3}{|c|}{ Earliest due datate } & \multicolumn{3}{|c|}{ Original due date } & \multicolumn{3}{|c|}{ Latest due date } \\
\hline & $\mathrm{ME}$ & MT & MAD & $\mathrm{ME}$ & MT & MAD & $\mathrm{ME}$ & MT & MAD \\
\hline ODD & 52.83 & $0.85^{*}$ & 53.69 & 52.88 & 0.81 & 53.69 & 53.21 & 0.74 & 53.95 \\
\hline JSLACK & 51.47 & 1.03 & 52.50 & 52.08 & 0.81 & 52.89 & 52.12 & 0.84 & 52.96 \\
\hline OSLACK & 51.77 & 1.00 & 52.77 & 52.07 & 0.84 & 52.91 & 51.79 & 0.96 & 52.76 \\
\hline MOD & 54.02 & 1.25 & 55.27 & 53.33 & 0.78 & 54.11 & 53.41 & $0.69^{*}$ & 54.10 \\
\hline MDD & 53.71 & 1.76 & 55.47 & 53.69 & 1.57 & 55.27 & 53.71 & 1.43 & 55.14 \\
\hline \multicolumn{10}{|l|}{ New rules } \\
\hline NJS & 45.50 & 2.20 & 47.70 & 46.54 & 3.70 & 50.24 & 47.48 & 6.57 & 54.04 \\
\hline NOS & 48.62 & 1.71 & 50.33 & 48.58 & 1.51 & 50.09 & 47.97 & 2.51 & 50.47 \\
\hline MJS & $45.44^{\prime \prime}$ & 1.8 & $47.24^{\prime \prime}$ & $45.44^{\prime \prime}$ & 1.8 & $47.24^{\prime \prime}$ & $45.44^{\prime \prime}$ & 1.8 & $47.24^{\prime \prime}$ \\
\hline MOS & 48.31 & 0.89 & 49.19 & 48.31 & 0.89 & 49.19 & 48.31 & 0.89 & 49.19 \\
\hline
\end{tabular}

due date information category, the MAD performance of due date based rules versus the due date information categories' graphs are also plotted in Figs. 2-5. From these figures, we observe that, with the exception of MOD, all other rules produce the same performance for all due date information used. This concludes that, in general, the rules are quite robust to the due date information used for the MAD measure. In fact, the robustness of rules like EDD and ODD is mainly due to the nature of the due windows assigned. Since the earliest and latest due dates are almost marginal values of the original due date, the relative ranking of the jobs due to EDD or ODD remains almost the same, irrespective of the nature of the due date.

On the other hand, exceptionally, MOD performs differently for each due date information used, and in general gives the best MAD performance when the latest due date is used. Note that MOD is a combinatorial rule that uses operation due date rule (ODD) until jobs get tardy and then substitutes ODD for the shortest processing time rule (SPT). Consequently, when latest due date is used, ODD is used more than SPT, than when earliest or original due date are taken into account. The fact that ODD performs better than SPT for the MAD measure can explain why MOD simply performs better when the latest due date is used. MDD has also the tendency to behave similarly to MOD, but surprisingly this behaviour is hardly observed in Table 3. This may be due to the fact that MDD utilizes the job rather than operation based information. On the other hand, we further observe that OSLACK and JSLACK are just robust, despite any experimental condition.

\subsection{Results of the proposed rules}

In this section, we will have a close look at the performance of the proposed rules NOS, NJS, MOS, and MJS, with a major focus on the latter pair of rules. The first pair of rules (i.e. NOS and NJS) are included in the study just to have a supportive role to the development process of our two new rules MOS and MJS. The reason for this is explained by what follows; according to the simulation results as figured in Table 3, we can make the following observations:

- When the earliest due date is used, NJS displays the best MAD performance with loose due dates; nevertheless, its performance deteriorates dramatically as either the original or the latest due date is used.

- NOS performance in terms of MAD shows to be robust to the nature of the due date information, but in almost all conditions, it performs no better than the best performing traditional rules.

The two observations above give us in brief the main drawbacks of the NOS and NJS: that is, even though they seem to have the right structure to overcome any other rule in terms of MAD, experimentally, they prove not to be so. Consequently, we feel ourselves pushed to seek better structured and performing rules like MOS and MJS, whose performance is analyzed in what follows.

By examining the results in Table 3, we observe that MOS displays the best MAD values, especially at high utilization rates. But, in the loose due date's case, either at high or low utilization rates, MJS gives best performance. This clearly proves the superiority of the new rules over the other ten well-known rules for the MAD measure. Furthermore, we note that the new rules are also effective in reducing $\mathrm{ME}$; according to Table 3, MJS always gives the best ME value under almost all experimental conditions. MOS shows also better ME performance than all the other rules (except MJS). In terms of the variability of the prediction error, the two new rules perform well, especially in the tight due date cases (Table 4). We also note that MJS is better than MOS as it displays lower standard deviation values for each measure (i.e. earliness, tardiness and mean absolute deviation).

In addition, MOS and MJS have the advantage of 
Table 4

Standard deviation of earliness (SE), tardiness (ST) and absolute deviation (SAD) simulation results

\begin{tabular}{|c|c|c|c|c|c|c|c|c|c|}
\hline \multicolumn{3}{|c|}{ Earliest due date } & \multicolumn{3}{|c|}{ Original due date } & \multicolumn{4}{|c|}{ Latest due date } \\
\hline & $\mathrm{SE}$ & ST & SAD & SE & ST & SAD & SE & ST & SAD \\
\hline \multicolumn{10}{|c|}{ High utilisation $(85 \%)$ /Tight due dates $(k=3.8)$} \\
\hline SPT & 8.14 & 21.12 & 13.93 & 8.14 & 21.12 & 13.93 & 8.14 & 21.12 & 13.93 \\
\hline STPT & 4.37 & 31.59 & 27.58 & 4.37 & 31.59 & 27.58 & 4.37 & 31.59 & 27.58 \\
\hline FCFS & 11 & 33.91 & 24.13 & 11 & 33.91 & 24.13 & 11 & 33.91 & 24.13 \\
\hline FAFS & 14.55 & 30.07 & 17.42 & 14.55 & 30.07 & 17.42 & 14.55 & 30.07 & 17.42 \\
\hline EDD & 12.27 & 23.06 & 14.5 & 11.76 & 23.41 & 14.09 & 13.05 & 24.41 & 14.54 \\
\hline ODD & 13.86 & 22.87 & 12.97 & 13.38 & 23.07 & 13.57 & 13.97 & 25.04 & 15.11 \\
\hline JSLACK & 12.39 & 26.99 & 17.83 & 12.66 & 24.63 & 15.21 & 12.94 & 25.50 & 15.08 \\
\hline OSLACK & 12.41 & 25.99 & 17.36 & 12.30 & 24.18 & 15.96 & 13.68 & 25.36 & 16.03 \\
\hline MOD & 11.37 & 17.12 & 8.6 & 11.08 & 16.57 & 8.16 & 11.36 & 15.41 & 8.43 \\
\hline MDD & 11.52 & 18.89 & 8.39 & 12.25 & 20.56 & 10.29 & 12.37 & 20.75 & 10.81 \\
\hline \multicolumn{10}{|l|}{ New rules } \\
\hline NJS & 8.78 & 22.18 & 15.49 & 8.81 & 20.73 & 14.02 & 9.74 & 22.54 & 14.18 \\
\hline NOS & 7.53 & 17.85 & 11.04 & 7.85 & 18.44 & 11.42 & 8.16 & 17.84 & 11.91 \\
\hline MJS & 1.15 & 8.71 & 9.86 & 1.15 & 8.71 & 9.86 & 1.15 & 8.71 & 9.86 \\
\hline MOS & 9.3 & 16.56 & 10.11 & 9.3 & 16.56 & 10.11 & 9.3 & 16.56 & 10.11 \\
\hline \multicolumn{10}{|c|}{ High utilisation $(85 \%) /$ Loose due dates $(k=6.5)$} \\
\hline SPT & 15.07 & 12.27 & 7.17 & 15.07 & 12.27 & 7.17 & 15.07 & 12.27 & 7.17 \\
\hline STPT & 10.47 & 23.11 & 15.99 & 10.47 & 23.11 & 15.99 & 10.47 & 23.11 & 15.99 \\
\hline FCFS & 34.84 & 8.74 & 26.92 & 34.84 & 8.74 & 26.92 & 34.84 & 8.74 & 26.92 \\
\hline FAFS & 35.64 & 10.76 & 25.38 & 35.64 & 10.76 & 25.38 & 35.64 & 10.76 & 25.38 \\
\hline EDD & 36.38 & 0.58 & 35.94 & 37.86 & 0.37 & 37.61 & 33.99 & 0.55 & 33.56 \\
\hline ODD & 36.73 & 1.06 & 36.09 & 35.63 & 0.71 & 35.27 & 35.32 & 0.7 & 34.99 \\
\hline JSLACK & 41.16 & 0.58 & 40.77 & 38.45 & 0.27 & 38.27 & 38.00 & 0.28 & 37.83 \\
\hline OSLACK & 35.62 & 1.23 & 34.76 & 36.50 & 1.06 & 35.92 & 34.01 & 0.99 & 33.52 \\
\hline MOD & 35.63 & 2.62 & 33.96 & 35.27 & 1.3 & 34.62 & 34.36 & 0.84 & 33.94 \\
\hline MDD & 38.21 & 1.61 & 37.06 & 33.73 & 0.49 & 33.36 & 33.77 & 0.64 & 33.3 \\
\hline \multicolumn{10}{|l|}{ New rules } \\
\hline NJS & 35.47 & 1.71 & 34.18 & 35.84 & 3.02 & 33.26 & 33.49 & 7.31 & 26.52 \\
\hline NOS & 29.22 & 4.17 & 26.45 & 31.90 & 3.99 & 29.96 & 33.52 & 3.95 & 31.07 \\
\hline MJS & 14.14 & 0.67 & 14.82 & 14.14 & 0.67 & 14.82 & 14.14 & 0.67 & 14.82 \\
\hline MOS & 35.46 & 1.26 & 34.76 & 35.46 & 1.26 & 34.76 & 35.46 & 1.26 & 34.76 \\
\hline \multicolumn{10}{|c|}{ Low utilisation $(60 \%) /$ Tight due dates $(k=1.8)$} \\
\hline SPT & 1.31 & 3.68 & 2.66 & 1.31 & 3.68 & 2.66 & 1.31 & 3.68 & 2.66 \\
\hline STPT & 1.24 & 5.8 & 4.72 & 1.24 & 5.8 & 4.72 & 1.24 & 5.8 & 4.72 \\
\hline FCFS & 1.2 & 5.67 & 4.82 & 1.2 & 5.67 & 4.82 & 1.2 & 5.67 & 4.82 \\
\hline FAFS & 1.47 & 5.29 & 4.08 & 1.47 & 5.29 & 4.08 & 1.47 & 5.29 & 4.08 \\
\hline EDD & 1.31 & 5.13 & 4.14 & 1.14 & 5.31 & 4.55 & 1.33 & 5.54 & 4.63 \\
\hline ODD & 1.09 & 4.81 & 4.03 & 1.22 & 4.53 & 3.78 & 1.19 & 4.61 & 3.87 \\
\hline JSLACK & 1.13 & 4.68 & 3.85 & 1.03 & 5.03 & 4.30 & 1.31 & 4.72 & 3.63 \\
\hline OSLACK & 1.25 & 5.33 & 4.54 & 0.93 & 5.15 & 4.49 & 0.99 & 5.12 & 4.42 \\
\hline MOD & 1.27 & 3.48 & 2.45 & 1.31 & 3.59 & 2.83 & 1.24 & 3.3 & 2.63 \\
\hline MDD & 1.04 & 5.44 & 4.58 & 0.98 & 4.49 & 3.77 & 1.27 & 5.07 & 4.2 \\
\hline \multicolumn{10}{|l|}{ New rules } \\
\hline NJS & 1.37 & 4.09 & 3.17 & 1.14 & 3.80 & 2.95 & 1.07 & 4.02 & 3.18 \\
\hline NOS & 1.04 & 3.14 & 2.44 & 1.16 & 3.39 & 2.66 & 1.20 & 3.54 & 2.83 \\
\hline MJS & 1.45 & 1.80 & 3.25 & 1.45 & 1.80 & 3.25 & 1.45 & 1.80 & 3.25 \\
\hline MOS & 0.94 & 3.51 & 2.93 & 0.94 & 3.51 & 2.93 & 0.94 & 3.51 & 2.93 \\
\hline \multicolumn{10}{|c|}{ Low utilisation $(60 \%) /$ Loose due dates $(k=2.7)$} \\
\hline SPT & 3.33 & 1.58 & 2.24 & 3.33 & 1.58 & 2.24 & 3.33 & 1.58 & 2.24 \\
\hline STPT & 3.69 & 3.23 & 2.05 & 3.69 & 3.23 & 2.05 & 3.69 & 3.23 & 2.05 \\
\hline FCFS & 3.86 & 2.44 & 2.74 & 3.86 & 2.44 & 2.74 & 3.86 & 2.44 & 2.74 \\
\hline FAFS & 4.18 & 2.2 & 2.46 & 4.18 & 2.2 & 2.46 & 4.18 & 2.2 & 2.46 \\
\hline EDD & 4.39 & 1.25 & 3.58 & 4.65 & 1.84 & 3.76 & 4.67 & 1.31 & 4.11 \\
\hline
\end{tabular}


Table 4 (continued)

\begin{tabular}{|c|c|c|c|c|c|c|c|c|c|}
\hline \multicolumn{3}{|c|}{ Earliest due date } & \multicolumn{3}{|c|}{ Original due date } & \multicolumn{4}{|c|}{ Latest due date } \\
\hline & SE & ST & SAD & SE & ST & SAD & $\mathrm{SE}$ & ST & SAD \\
\hline ODD & 3.82 & 0.72 & 3.47 & 3.76 & 0.82 & 3.4 & 3.71 & 0.67 & 3.37 \\
\hline JSLACK & 4.27 & 1.13 & 3.58 & 4.64 & 0.90 & 4.18 & 4.45 & 0.90 & 4.07 \\
\hline OSLACK & 3.84 & 0.80 & 3.40 & 3.96 & 0.77 & 3.53 & 4.21 & 0.84 & 3.68 \\
\hline MOD & 2.63 & 0.95 & 2.05 & 3.7 & 0.63 & 3.36 & 3.9 & 0.67 & 3.58 \\
\hline MDD & 4.4 & 1.51 & 3.43 & 4.59 & 1.78 & 3.63 & 5.04 & 1.37 & 4.36 \\
\hline \multicolumn{10}{|l|}{ New rules } \\
\hline NJS & 4.13 & 1.15 & 3.30 & 3.53 & 1.53 & 2.62 & 3.73 & 2.05 & 2.06 \\
\hline NOS & 4.15 & 1.17 & 3.47 & 3.70 & 0.82 & 3.23 & 3.63 & 1.19 & 2.98 \\
\hline MJS & 1.29 & 1.73 & 3.03 & 1.29 & 1.73 & 3.03 & 1.29 & 1.73 & 3.03 \\
\hline MOS & 3.58 & 0.77 & 3.18 & 3.58 & 0.77 & 3.18 & 3.58 & 0.77 & 3.18 \\
\hline
\end{tabular}

High Utilization $(85 \%) /$ Tight due-dates $(\mathrm{k}=3.8)$
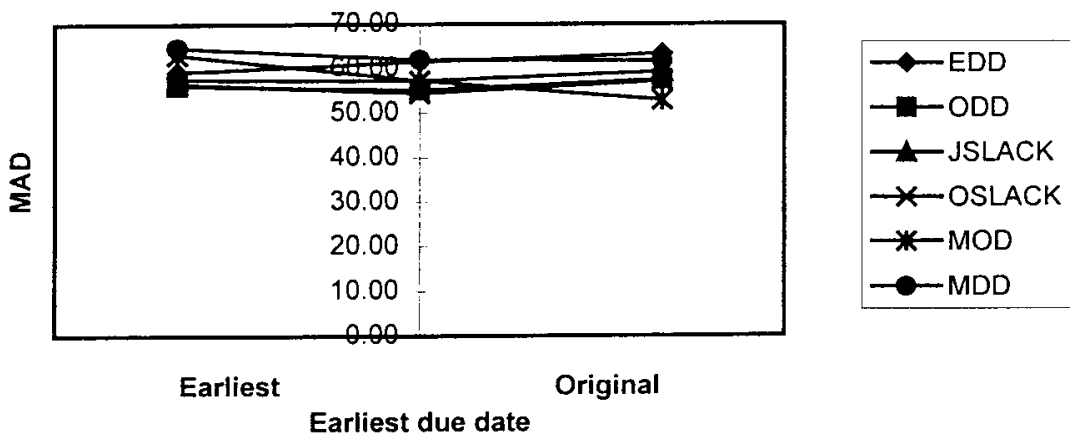

Fig. 2. MAD vs due date information/exp. cond. 1.

High utilization (85\%) / Loose due dates ( $k=6.5$ )

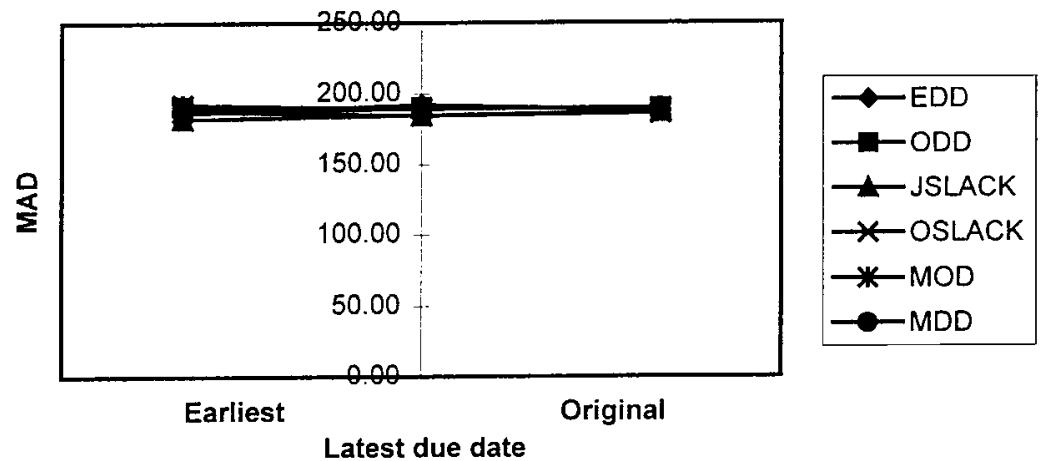

Fig. 3. MAD vs due date information/exp. cond. 2. 


\section{Low utilization $(60 \%)$ / Tight due dates $(k=1.8)$}
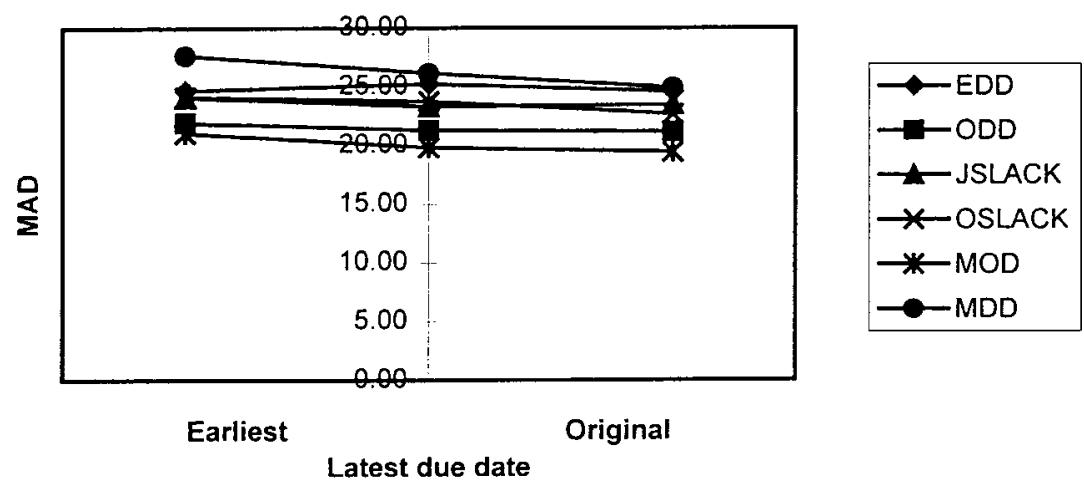

Fig. 4. MAD vs due date information/exp. cond. 3 .

using multi due date information. Their structure enables them to consider two due date information (earliest and latest due dates) that are assumed to be representative of the due window. Furthermore, these rules are simple to implement in job shop systems. They require no parameters to be estimated, other than using already available job information. This makes us more confident in suggesting MOS and MJS as two effective priority rules to achieve satisfactory MAD and ME performances in dynamic job shop environments.

Another important observation that emerged from our experiments is that operation-based rules are not necessarily more effective than job based rules. This is contrary to what is known in the literature for regular performance measures such as mean tardiness. In the non regular performance measure (i.e. MAD) case, however, we noted that MJS outperforms MOS for MAD in the loose due date's case, and for ME under all experimental conditions.

\subsubsection{The relative performance of $M O D, M J S$ and} MOS with different $R$ values

In this section, we extend our simulation experiments by measuring the performance of our two new rules as well as the most competing rule MOD, with respect to increasing due window sizes. Keeping all other conditions the same, we change the $R$ value from $0 \%$ to 10,20 and $\$ 40 \%$. Note that the due date tightness coefficient $k$ is adjusted according to the $0 \%$ level (i.e. no due window) and kept the same for the 10, 20 and $40 \%$ levels. The adjusted $k$ values as well as the results of the experimentation are illustrated in Table 5. Again, the pair $t$-tests are applied to measure the

\section{Low utilization $(60 \%)$ / Loose due dates $(k=2.7)$}
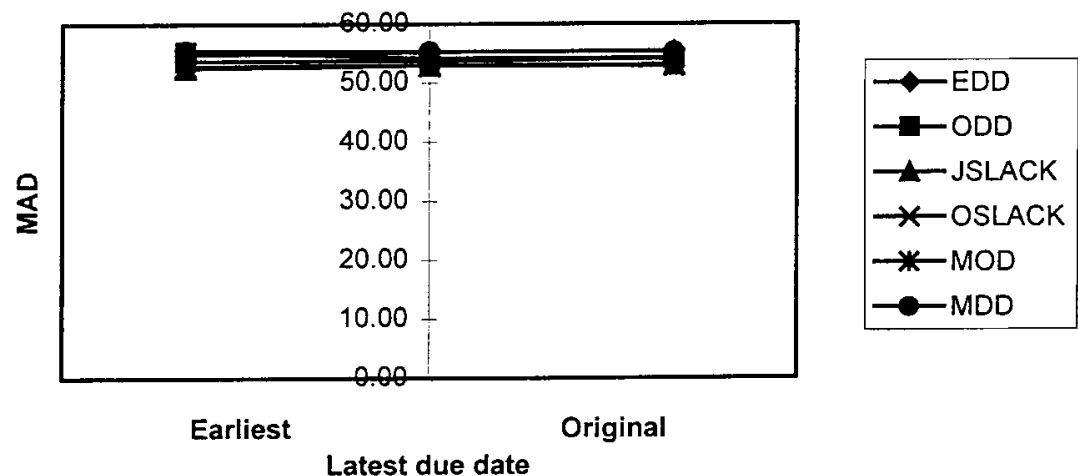

Fig. 5. MAD vs due date information/exp. cond. 4 . 
Table 5

ME, MT and MAD results for different $R$ values

\begin{tabular}{|c|c|c|c|c|c|c|c|c|c|}
\hline \multicolumn{3}{|c|}{ With earliest due date } & \multicolumn{3}{|c|}{ With original due date } & \multicolumn{4}{|c|}{ With latest due date } \\
\hline & $\mathrm{ME}$ & MT & MAD & ME & MT & MAD & $\mathrm{ME}$ & MT & MAD \\
\hline \multicolumn{10}{|c|}{$\begin{array}{l}\text { High utilization }(85 \%) / \text { Tight due dates }(k=4.5) \\
R=0 \%\end{array}$} \\
\hline MOD & 96.77 & $13.13^{*}$ & 109.90 & 96.77 & $13.13^{*}$ & 109.90 & 96.77 & $13.13^{*}$ & 109.90 \\
\hline MOS & 73.16 & 16.85 & 90.01 & 73.16 & 16.85 & 90.01 & 73.16 & 16.85 & 90.01 \\
\hline MJS & $59.21^{*}$ & 26.50 & $85.71^{*}$ & $59.21^{*}$ & 26.50 & $85.71^{*}$ & $59.21^{*}$ & 26.50 & $85.71^{*}$ \\
\hline \multicolumn{10}{|c|}{$R=10 \%$} \\
\hline MOD & 69.46 & 12.13 & 81.59 & 66.88 & 10.00 & 76.88 & 66.10 & 8.65 & 74.75 \\
\hline MOS & 47.43 & $9.90^{*}$ & 57.33 & 47.43 & 9.90 & 57.33 & 47.43 & 9.90 & 57.33 \\
\hline MJS & $42.08^{*}$ & 13.83 & 55.91 & $42.08^{*}$ & 13.83 & 55.91 & $42.08^{*}$ & 13.83 & 55.91 \\
\hline \multicolumn{10}{|c|}{$R=20 \%$} \\
\hline MOD & 46.50 & 14.41 & 60.91 & 43.46 & 8.20 & 51.66 & 45.95 & 5.24 & 51.19 \\
\hline MOS & 31.06 & $5.82^{*}$ & 36.88 & 31.06 & $5.82^{*}$ & 36.88 & 31.06 & 5.82 & 36.88 \\
\hline MJS & $27.83^{*}$ & 7.40 & 35.23 & $27.83^{*}$ & 7.40 & 35.23 & $27.83^{*}$ & 7.40 & 35.23 \\
\hline \multicolumn{10}{|c|}{$R=40 \%$} \\
\hline MOD & 15.43 & 16.44 & 31.87 & 14.93 & 5.95 & 20.88 & 18.62 & 1.89 & 20.51 \\
\hline MOS & 11.00 & 3.25 & 14.25 & 11.00 & 3.25 & 14.25 & 11.00 & 3.25 & 14.25 \\
\hline MJS & 10.70 & 2.72 & 13.42 & 10.70 & $2.72^{*}$ & 13.42 & 10.70 & 2.72 & 13.42 \\
\hline \multicolumn{10}{|c|}{ High utilization $(85 \%) /$ Loose due dates $(k=7)$} \\
\hline \multicolumn{10}{|c|}{$R=0 \%$} \\
\hline MOD & 283.01 & $0.51^{*}$ & 283.52 & 283.01 & $0.51^{*}$ & 283.52 & 283.01 & $0.51^{*}$ & 283.52 \\
\hline MOS & 228.70 & 1.13 & 229.84 & 228.70 & 1.13 & 229.84 & 228.70 & 1.13 & 229.84 \\
\hline MJS & $204.78^{*}$ & 2.16 & $206.94^{*}$ & $204.78^{*}$ & 2.16 & $206.94^{*}$ & $204.78^{*}$ & 2.16 & $206.94^{*}$ \\
\hline \multicolumn{10}{|c|}{$R=10 \%$} \\
\hline MOD & 224.22 & 0.77 & 224.99 & 224.98 & 0.27 & 225.25 & 222.48 & $0.16^{*}$ & 222.64 \\
\hline MOS & 175.59 & 0.27 & 175.86 & 175.59 & 0.27 & 175.86 & 175.59 & 0.27 & 175.86 \\
\hline MJS & $152.32^{*}$ & 0.24 & $152.56^{*}$ & $152.32 *$ & 0.24 & $152.56^{*}$ & $152.32^{*}$ & 0.24 & $152.56^{*}$ \\
\hline \multicolumn{10}{|c|}{$R=20 \%$} \\
\hline MOD & 173.55 & 1.28 & 174.83 & 170.45 & 0.18 & 170.64 & 169.32 & 0.05 & 169.37 \\
\hline MOS & 126.07 & 0.13 & 126.20 & 126.07 & 0.13 & 126.20 & 126.07 & 0.13 & 126.20 \\
\hline MJS & $113.21^{*}$ & $0.04^{*}$ & $113.25^{*}$ & $113.21^{*}$ & $0.04^{*}$ & $113.25^{*}$ & $113.21^{*}$ & 0.04 & $113.25^{*}$ \\
\hline \multicolumn{10}{|c|}{$R=40 \%$} \\
\hline MOD & 78.70 & 3.30 & 82.00 & 80.73 & 0.07 & 80.80 & 88.54 & 0.01 & 88.54 \\
\hline MOS & 58.36 & 0.06 & 58.42 & 58.36 & 0.06 & 58.42 & 58.36 & 0.06 & 58.42 \\
\hline MJS & $54.33^{*}$ & $0.01^{*}$ & $54.33^{*}$ & $54.33^{*}$ & $0.01^{*}$ & $54.33^{*}$ & $54.33^{*}$ & 0.01 & $54.33^{*}$ \\
\hline \multicolumn{10}{|c|}{ Low utilization $(60 \%) /$ Tight due dates $(k=2)$} \\
\hline \multicolumn{10}{|c|}{$R=0 \%$} \\
\hline MOD & 26.15 & $9.74^{*}$ & 35.89 & 26.15 & $9.74^{*}$ & 35.89 & 26.15 & $9.74^{*}$ & 35.89 \\
\hline MOS & 22.87 & 12.22 & 35.09 & 22.87 & 12.22 & 35.09 & 22.87 & 12.22 & 35.09 \\
\hline MJS & $22.06^{*}$ & 15.62 & 37.68 & $22.06^{*}$ & 15.62 & 37.68 & $22.06^{*}$ & 15.62 & 37.68 \\
\hline \multicolumn{10}{|c|}{$R=10 \%$} \\
\hline MOD & 16.66 & 7.15 & 23.81 & 15.71 & 6.25 & 21.96 & 15.83 & 5.85 & 21.68 \\
\hline MOS & 14.23 & 6.70 & 20.93 & 14.23 & 6.70 & 20.93 & 14.23 & 6.70 & 20.93 \\
\hline MJS & 13.79 & 9.54 & 23.33 & 13.79 & 9.54 & 23.33 & 13.79 & 9.54 & 23.33 \\
\hline \multicolumn{10}{|c|}{$R=20 \%$} \\
\hline MOD & 9.13 & 5.73 & 14.86 & 8.40 & 4.40 & 12.80 & 8.86 & 3.51 & 12.37 \\
\hline MOS & 7.80 & 4.07 & $11.87^{*}$ & 7.80 & 4.07 & 11.87 & 7.80 & 4.07 & 11.87 \\
\hline MJS & 7.69 & 5.60 & 13.30 & 7.69 & 5.60 & 13.30 & 7.69 & 5.60 & 13.30 \\
\hline \multicolumn{10}{|c|}{$R=40 \%$} \\
\hline MOD & 1.11 & 4.34 & 5.45 & 1.01 & 2.48 & 3.49 & 1.05 & $1.13^{*}$ & 2.18 \\
\hline MOS & 1.04 & 1.94 & 2.98 & 1.04 & 1.94 & 2.98 & 1.04 & 1.94 & 2.98 \\
\hline MJS & 1.00 & $1.76^{*}$ & $2.76^{*}$ & 1.00 & $1.76^{*}$ & $2.76^{*}$ & 1.00 & 1.76 & 2.76 \\
\hline Utiliza & \%)/Loose & dates $(k$ & & & & & & & \\
\hline$R=0$ & & & & & & & & & \\
\hline MOD & 98.09 & $0.66^{*}$ & 98.75 & 98.09 & $0.66^{*}$ & 98.75 & 98.09 & $0.66^{*}$ & 98.75 \\
\hline
\end{tabular}


Table 5 (continued)

\begin{tabular}{|c|c|c|c|c|c|c|c|c|c|}
\hline \multicolumn{3}{|c|}{ With earliest due date } & \multicolumn{3}{|c|}{ With original due date } & \multicolumn{4}{|c|}{ With latest due date } \\
\hline & ME & MT & MAD & $\mathrm{ME}$ & MT & MAD & ME & MT & MAD \\
\hline MOS & 90.09 & 1.08 & 91.17 & 90.09 & 1.08 & 91.17 & 90.09 & 1.08 & 91.17 \\
\hline MJS & $85.59^{*}$ & 2.33 & $87.92^{*}$ & $85.59^{*}$ & 2.33 & $87.97^{*}$ & $85.59^{*}$ & 2.33 & $87.92^{*}$ \\
\hline \multicolumn{10}{|c|}{$R=10 \%$} \\
\hline MOD & 74.33 & 0.49 & 74.82 & 73.63 & 0.37 & 74.00 & 73.49 & $0.28^{*}$ & 73.77 \\
\hline MOS & 66.69 & 0.45 & 67.14 & 66.69 & 0.45 & 67.14 & 66.69 & 0.45 & 67.14 \\
\hline MJS & $64.26^{*}$ & 0.85 & $65.10^{*}$ & $64.26^{*}$ & 0.85 & $65.10^{*}$ & $64.26^{*}$ & 0.85 & $65.10^{*}$ \\
\hline \multicolumn{10}{|c|}{$R=20 \%$} \\
\hline MOD & 52.00 & 0.70 & 52.70 & 51.16 & 0.24 & 51.40 & 51.69 & 0.16 & 51.85 \\
\hline MOS & 46.40 & 0.21 & 46.61 & 46.40 & 0.21 & 46.61 & 46.40 & 0.21 & 46.61 \\
\hline MJS & $44.35^{*}$ & 0.27 & $44.63^{*}$ & $44.35^{*}$ & 0.27 & $44.63^{*}$ & $44.35^{*}$ & 0.27 & $44.63^{*}$ \\
\hline \multicolumn{10}{|c|}{$R=40 \%$} \\
\hline MOD & 16.66 & 0.99 & 17.65 & 16.69 & 0.12 & 16.81 & 17.24 & 0.05 & 17.30 \\
\hline MOS & 15.41 & 0.10 & 15.51 & 15.41 & 0.10 & 15.51 & 15.41 & 0.10 & 15.51 \\
\hline MJS & $14.76^{*}$ & 0.09 & $14.85^{*}$ & $14.76^{*}$ & 0.09 & $14.85^{*}$ & $14.76^{*}$ & 0.09 & $14.85^{*}$ \\
\hline
\end{tabular}

significance of the difference of the best two rules performances. The sign $\left(^{*}\right)$ indicates that the difference is significant at $\alpha=0.05$ level.

According to Table 5, we observe mainly that as the radius coefficient increase, the performance of the rules improves. More importantly, the improvement is more magnified for the two new rules MOS and MJS. This means that, as the due window size increases, the relative performance of MOS and MJS gets better than MOD.

Another point worth noting is that, MOD gives different performances with different due date information and eventually performs best with the latest due date in all experimental conditions. Interestingly, this behaviour is more magnified as the window size gets larger, which confirms the discussion done pre- viously to explain the lack of robustness of MOD to the nature of the due date information.

\subsubsection{The extremely tight due dates case}

In light of the symmetric criterion MAD, the new four rules, as well as the most competing rule MOD, and FCFS as a benchmark rule, are tested with extremely tight due dates, where $90 \%$ PT (equivalently $10 \%$ proportion of early jobs) is considered. The $k$ value is adjusted accordingly with the FCFS rule. Illustration of the $k$ values, as well as the results for ME, MT, and MAD are presented in a similar tabular format as before, in Table 6 .

According to Table 6, we observe that MOD displays the best performance in all conditions, closely followed by NOS, NJS, MOS, and MJS. Interestingly,

Table 6

Performance of the rules with very tight due dates $(90 \%$ PT)

\begin{tabular}{|c|c|c|c|c|c|c|c|c|c|}
\hline & \multicolumn{3}{|c|}{ Earliest due date } & \multicolumn{3}{|c|}{ Original due date } & \multicolumn{3}{|c|}{ Latest due date } \\
\hline & ME & MT & MAD & ME & MT & MAD & ME & MT & MAD \\
\hline \multicolumn{10}{|c|}{ High utilization $(85 \%) /$ very tight due dates $k=1.6$} \\
\hline FCFS & 0.46 & 193.12 & 193.58 & 0.46 & 193.12 & 193.58 & 0.46 & 193.12 & 193.58 \\
\hline MOD & 0.72 & $114.52^{*}$ & $115.2^{*}$ & 0.57 & 118.41 & 118.98 & 0.56 & $116.14^{*}$ & $116.70^{*}$ \\
\hline NJS & 0.45 & 123.22 & 123.67 & 0.45 & 128.77 & 129.22 & 0.48 & 134.03 & 134.51 \\
\hline NOS & 0.53 & 118.18 & 118.71 & 0.54 & 119.12 & 119.66 & 0.50 & 120.51 & 121.01 \\
\hline MJS & 0.48 & 147.18 & 147.66 & 0.48 & 147.18 & 147.66 & 0.48 & 147.18 & 147.66 \\
\hline MOS & 0.43 & 142.54 & 142.97 & 0.43 & 142.54 & 142.97 & 0.43 & 142.54 & 142.97 \\
\hline \multicolumn{10}{|c|}{ Low utilization $(60 \%) /$ very tight due dates $k=1$} \\
\hline FCFS & 0.00 & 71.29 & 71.29 & 0.00 & 71.29 & 71.29 & 0.00 & 71.29 & 71.29 \\
\hline MOD & 0.00 & 55.82 & 55.82 & 0.00 & 55.82 & 55.82 & 0.00 & 55.41 & 55.41 \\
\hline NJS & 0.00 & 55.82 & 55.82 & 0.00 & 55.82 & 55.82 & 0.00 & 57.31 & 57.31 \\
\hline NOS & 0.00 & 55.82 & 55.82 & 0.00 & 55.82 & 55.82 & 0.00 & 55.89 & 55.89 \\
\hline MJS & 0.00 & 60.72 & 60.72 & 0.00 & 60.72 & 60.72 & 0.00 & 60.72 & 60.72 \\
\hline MOS & 0.00 & 60.60 & 60.60 & 0.00 & 60.60 & 60.60 & 0.00 & 60.60 & 60.60 \\
\hline
\end{tabular}


MOD, NOS, and NJS produce the same performance in the low utilization case when either the earliest or the original due date is used. This can be explained by the fact that, in these particular conditions, the three rules reduce to the SPT rule, a consequence of the fact that all jobs are tardy. Finally, we conclude our remarks by stating that our rules of concern, MOS and MJS may not be the most appropriate rules for MAD, under such extremely tight due dates cases, where tardiness based rules show to be the most effective. Intuitively, this is expected since in such conditions, minimizing MAD is just equivalent to minimizing MT.

\section{Conclusion}

In this paper, we investigated the performance of well-known dispatching rules in terms of the non regular measure (MAD) under the due window approach. The results indicate that the rules, which are known to be very effective for completion time and tardiness based criteria, are not appropriately for MAD. Hence, we tested four new rules, among which only one pair is proposed. The main findings of our study are as follows:

1. Processing time based rules such as SPT, STPT are not appropriate for MAD, since they have the tendency to maximize earliness instead of minimizing it.

2. EDD, ODD, JSLACK, OSLACK, MOD and MDD show better MAD performances than SPT and STPT. Consequently, they fit more appropriate for MAD. Nevertheless, their performance is poor in the loose due date's case due to the high ME values.

3. Except MOD, due date based rules are quite robust to the due date information used. This avoids the difficulty of selecting the due date information when applying the rules with the due window approach.

4. The proposed two rules-MOS and MJS - are more effective to minimize MAD as well as ME than the other ten existing rules, except under the extremely tight due dates case. Furthermore, their efficiency increases as the due window size gets larger. These rules are not only simple to implement in dynamic job shop environments but also their structure enables us to consider multi due date information.

\section{References}

[1] Bagchi U, Chang Y, Sullivan R. Minimizing absolute and squared deviations of completion times with different earliness and tardiness penalties and a common due date. Naval Res Logist Quart 1987;34:739-41.

[2] Baker KR, Kanet JJ. Job shop Scheduling with modified due dates. J Ops Mgmt 1983;4(1):11-22.

[3] Baker KR, Scudder GD. Sequencing with earliness and tardiness penalties: A review. Ops Res 1990;38:22-36.

[4] Baker KR. Sequencing rules and due date assignments in a job shop. Mgmt Sci 1984;30(9):1093-104.

[5] Conway RW, Maxwell WL. Network dispatching by the shortest operation discipline. Ops Res 1962;10:51-73.

[6] Emmons H. Scheduling to a common due date on parallel common processors. Naval Res Logist Quart 1987;34:803-10.

[7] Hall N. Single and multi-processor models for minimizing completion time variance. Naval Res Logist Quart 1986;33:49-54.

[8] Kanet JJ. Minimizing the average deviation of job completion times about a common due date. Naval Res Logist Quart 1981;28:643-51.

[9] Kiran AS, Smith ML. Simulation studies in job shop scheduling II: Performance of priority rules. Comp And Indust Engng 1984;8(2):95-105.

[10] Franz-Josef Krämer, Lee Chung-Yee. Common due window scheduling. Prod and Ops Mgmt 1993;2 (4):262-75.

[11] Law AM, Kelton WD. Simulation Modeling and Analysis. McGraw-Hill, 1991.

[12] Pegden CD, Shannon RE, Sadowsky RP. Introduction to Simulation Using Siman. McGraw-Hill, 1995.

[13] Sundararaghavan P, Ahmet M. Minimizing the sum of absolute lateness in single machine and multimachine scheduling. Naval Res Logist Quart 1984;31:325-33.

[14] Vepsalainen APJ, Morton TE. Priority rules for job shops with weighted tardiness costs. Mgmt Sci 1987;33(8):1035-47.

[15] Weng Michael X, Ventura Jose A. Scheduling about a large common due date with tolerance to minimize mean absolute deviation of completion times. Naval Res Logists 1993;41(6):843-51. 